\title{
A NOTE ON INTEGRAL EQUATIONS
}

\author{
by W. E. WILLIAMS
}

(Received 20 August, 1970)

In a recent paper Cooke [1] obtained a solution of the integral equation

$$
\int_{0}^{1} g(t) \log \left|\frac{x+t}{x-t}\right| d t=\pi f(x) \quad(0<x<1)
$$

by using the identity

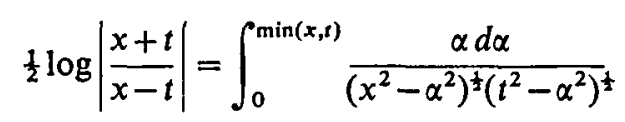

and the technique, first used by Copson, of interchanging the orders of integration and hence fucing the problem to that of the successive solution of two Abel integral equations. It is also shown in [1] that the above identity can also be used to solve the dual series equations

$$
\begin{array}{ll}
\sum_{n=1}^{\infty} \frac{a_{n}}{n} \sin n x=f(x) & (0<x<c), \\
\sum_{n=1}^{\infty} a_{n} \sin n x=0 & (c<x<\pi) .
\end{array}
$$

The kernel in equation (1) is a particular member of a general class of kernels which the author [6] has shown to be such that the resulting integral equation is directly soluble by using Copson's technique. The particular example of equation (1) is given in [6] and the identity of equation (2) was used by the author [7] to obtain the solution of equation (3).

It is also shown in [6] that the identity of equation (2) can be used to solve the " aerofoil " equation and the explicit solution is obtained. The form of solution obtained is not the usual one quoted for the solution of the aerofoil equation in that it does not involve a Cauchy principal value integral, but it can be reduced to the usual standard form. The same general approach was also used independently by Peters [3] to solve the aerofoil equation and it is interesting to note that the form of solution obtained in references [6] and [3] is identical with one obtained by Cooke [1], who transformed Carleman's solution for an equation of the form

$$
\int_{a}^{b} g(t) \log |x-t| d t=\pi f(x)
$$

so as not to involve Cauchy principal value integrals. Differentiation of equation (4) produces the aerofoil equation and hence the solution of this latter equation may be found provided that equation (4) can be solved.

The transformation of an equation of the general form of equation (4) to a form amenable to solution by direct use of equation (2) was sketched in [6] but the explicit solution was not 
given. The most direct method of solving equation (4) seems to be to transform it by differentiation to the aerofoil equation and then employ the results of [6] or [3]. It follows immediately from equation, 13 , of [3] that the solution of equation (4) is

$$
g(t)=\frac{c}{(t-a)^{\frac{1}{2}}(b-t)^{\frac{1}{2}}}+\frac{1}{\pi} \int_{t}^{b}(\sigma-t)^{-\frac{1}{2}} \int_{a}^{\sigma} \frac{(x-a)^{\frac{1}{2}}}{(\sigma-x)^{\frac{1}{2}}} f^{\prime}(x) d x d \sigma,
$$

where $c=\int_{a}^{b} g(t) d t$.

Equation (4) may be rewritten as

$$
\int_{-1}^{1} G(t) \log |x-t| d t=\frac{2 \pi F(x)}{b-a}-\log \left(\frac{b-a}{2}\right) \int_{-1}^{1} G(t) d t
$$

where $G(t)=g\left[\frac{1}{2}(b-a)(t+1)+a\right], F(x)=f\left[\frac{1}{2}(b-a)(x+1)+a\right]$. Equation (6) is of the form considered in [6]; from equation (28) of that paper, $\int_{-1}^{1} G(t) d t$ and hence $\int_{a}^{b} g(t) d t$ may be calculated, and we have that

$$
c=\int_{a}^{b} g(t) d t=-\frac{1}{[2 \log 2-\log (b-a)]} \int_{a}^{b} \frac{f(x)}{(x-a)^{\frac{1}{2}}(b-x)^{\frac{1}{2}}} d x .
$$

Equation (5) with $c$ defined as above agrees with the result derived by Cooke.

It was also pointed out in [6] that another simple example of the general class of kernel is the kernel $|x-t|^{-\alpha}$ and an explicit solution was given for the integral equation

$$
\int_{0}^{1} g(t)|x-t|^{-\alpha} d t=f(x) \quad(0<x<1,0<\alpha<1) .
$$

Equation (7) has also recently been solved by Lundgren and Chiang [2] by means of the Wiener-Hopf technique and they also applied this technique to the problem of solving the equation

$$
\int_{0}^{1} g(t) \operatorname{sgn}(x-t)|x-t|^{-\alpha} d t=f(x) \quad(0<x<1,0<\alpha<1) .
$$

Though the analysis of [2] relating to equation (7) and the representation of the kernel of [6] break down for $-1<\alpha<0$, the final form obtained for the solution is valid for $\alpha$ within this range and hence the solution of the equation

$$
\int_{0}^{1} g(t)|x-t|^{1-\alpha} d t=f(x)
$$

can be obtained from the solution of equation (7) on replacing $\alpha$ by $\alpha-1$. It can be shown that the solution so obtained agrees with that obtained by Lundgren and Chiang who, by differentiation, reduced equation (9) to the form of equation (8). The demonstration that the two forms of the solution are equivalent is not completely straightforward and is sketched in the Appendix.

Various generalisations of equations (7) and (8) to equations where the path of inte- 
gration is a curve in the complex plane have also recently been considered by Peters [5] and the reduction of singular Cauchy integral equations of the second type to the successive solution of two Abel equations has been presented by Peters in [4].

\section{APPENDIX}

The solution obtained for equation (9) by replacing $\alpha$ by $\alpha-1$ is given by $2 \Gamma(2-\alpha) \Gamma^{2}\left(\frac{1}{2} \alpha\right) \cos \frac{1}{2} \alpha \pi g(t)$

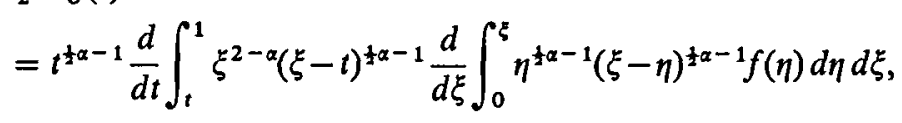

and that given by Lundgren and Chiang is

$$
\begin{aligned}
& 2 \Gamma(1-\alpha) \Gamma^{2}\left(\frac{1}{2} \alpha\right) \cos \alpha \pi g(t)=t^{-1+\frac{1}{2} \alpha}(1-t)^{-1+\frac{1}{2} \alpha} \int_{0}^{1} f(\beta) \beta^{\frac{1}{\alpha} \alpha-1}(1-\beta)^{\frac{1}{2} \alpha-1} d \beta \\
& +\frac{d}{d t} t^{\frac{1 \alpha}{2 \alpha}} \int_{t}^{1} \xi^{-\alpha}(\xi-t)^{\frac{1}{\alpha} \alpha-1} d \xi \int_{0}^{\xi} \frac{\beta^{\frac{1 \alpha}{\alpha}}(\xi-\beta)^{\frac{1 \alpha-1}{2}}}{(1-\alpha)} f^{\prime}(\beta) d \beta .
\end{aligned}
$$

It can be established by integration by parts that

$$
\begin{aligned}
& \int_{0}^{\xi} \beta^{\frac{1}{a} \alpha}(\xi-\beta)^{\frac{\xi}{\alpha}-1} f^{\prime} d \beta=\int_{0}^{\xi} \beta^{\frac{1}{\alpha^{\alpha}-1}}(1-\alpha)(\xi-\beta)^{\frac{1}{\alpha} \alpha-1} f d \beta+\xi \frac{d}{d \xi} \int_{0}^{\xi} \beta^{\frac{1}{2} \alpha-1}(\xi-\beta)^{\frac{1}{\alpha} \alpha-1} f d \beta, \\
& \int_{t}^{1} \xi^{1-\alpha}(\xi-t)^{t \alpha-1} H(\xi) d \xi=H(1)(1-t)^{\frac{t \alpha-1}{\alpha}} \\
& +t^{1-\frac{t a}{\alpha}} \frac{d}{d t}\left(t^{\frac{1}{2} \alpha} \int_{t}^{1} \xi^{-\alpha}(\xi-t)^{\frac{t^{\alpha}-1}{} H d \xi}\right) \quad(\alpha>0) .
\end{aligned}
$$

The equivalence of the two solutions can now be established by applying (A.3) to the inner integral in (A.2) and then applying (A.4) to the integral arising from the first term of (A.3).

\section{REFERENCES}

1. J. C. Cooke, The solution of some integral equations and their connection with dual integral equations and series, Glasgow Math. J. 11 (1970), 9-20.

2. T. Lundgren and D. Chiang, Solution of a class of singular integral equations, Quart. Appl. Math. 24 (1967), 303-313.

3. A. S. Peters, A note on the integral equation of the first kind with a Cauchy kernel, Comm. Pure App. Math. 16 (1963), 57-61.

4. A. S. Peters, Abel's equation and the Cauchy integral equation of the second kind, Comm. Pure App. Math. 21 (1968), 51-65.

5. A. S. Peters, Some integral equations related to Abel's equation and the Hilbert transform, Comm. Pure App. Math. 22 (1969), 539-560.

6. W. E. Williams, A class of integral equations, Proc. Cambridge Philos. Soc. 59 (1963), 589-597.

7. W. E. Williams, The solution of dual series and dual integral equations, Proc. Glasgow Math. Assoc. 6 (1964), 123-129.

UNIVERSITY OF SURREY 